Fragmenta, memoriam tum certam, tum latissimam suis auctoribus conciliatura, eam praeferre convenit naturam, ut colligantur, componantur, explanentur. Qua ipsa triplici necessitate quantis editor implicetur negotiis, non difficulter intelligi licet. Praestant enim auctores, quorum opera utcunque integra stetenunt, aro gumentum perspicuum, definitam quandam ratiocina. tionum seriem et complexum, decursum orationis in. stitutae reliquis aptum, ambitumque accuratis circumocriptum limitibus: ordinem denique perpetuum, ad quem etiam minutissiniae quaeque sententiae exigantut. At vero quorum virorum ingenii monumenta temporum sive iniuria sive potius cura posteritatem non at. tigerint, in obscuro argumento atque in ruinis versan. tur, neque luminibus ant verbornm mente prababili conclusorum, aut sententiartum omne propositum expes dientium, illustrantur. $\Delta c$ primum collectione reli. quiarum rem intligere apparet, quae ut clarissima viri fere ignoti effigies animo concipiatur, ne sublestissimum quidem dictum opinionemve praetermittat. Unde conficitur, lectionem requiri universos antiquitatis libros complexam. Est sane immensa ea eruditio non alia, quam diligentiae pertinacissimae laus et progenies; sed quotusquisque adeo et $a b$ otio et ab aevi amplitudine beatus reperitur, ut ad istud fastigitum evehi potuerit? Inaudivimus adhac de Triumviris Batavis, Hemsterhusio, Valckenario, et quem dubitanter adiicio Ruhnke- 
nio. Possit, neque immerito, ab indicibus auctorum (auctoribus enim praecipue significandis, ut quorum loci etiam per, accuratissima digesti adversaria quaerentem saepenumero lateant, index debebat addictus esse.) subsidii aliquid exspectari; verum isti nimia negligentia fere ubique constructi deprehenduntur. Proinde non facile quisquam unum alterumve fragmentum hic quoque omissum mirabitur, cum praesertim ne Bentleium quidem, hoc in genere administrando prae ceteris insignem, Callimacheorum nihil effugerit. Accerlit, nt eorum hominum, qui late patentem olim obtinuerint celebritatem, non pauca etiam apud eos quos minime exspectares auctores, nomine sive neglecto, sive depravato, latitent, qualia vix critici exercitatissimi acumen penitus eruerit.

Deinde fragmentis componendis collatorem sese addicere decet. Quod si interpretis praestantia, qui argumenti quamvis expositi, planam tamen intelligentiam non ab omni parte admittentis, molestias ita sustulerit, ut ordinem et nominum et sententiarnm usquequaque perspicuum commonstret et exhibeat, eius igitur interpretis praestantia iustissimis effertur laudibus : multo etiam maiore in loco ille ponendus erit, qui argumentum neque ad omnium cognitionem obiectum, neque ansam copiamque sui faciens, in integrum restituerit, et, lacunis quantumvis impedientibus, explicationem et ambitum proposuerit. Ex hac studiorum parte, omnium difficillima, plerosque fragmentorum edítores minus censeri voluisse, non utique est quod miremur: mireris tamen, illum, quem sagacitate perpauci aequipararunt, Bentleium, prorsus ignorasse videri, etiam Callimachi, reliquias concinnandas fuisse. Unde necessario accidit, ut quae dextre disposita adnmbrationem certe ingenii et mentis, qua locos suos auctor pertractare consuesset, forent constitutura, ea nonnisi curiosi- 
tati minutiarum studiosac satisfacere queant. Inde etiam aliud aliquanto gravius malum provenit, quod libros illi affingebant nunquam evulgatos, magnam istos partem vel indoli scriptoris admotos procul abiiciendos. Veluti ne inprimis novissimos huils negotii administratores ambitiose perstringam, eidem Callinuacho Criticus Britannus viginti ipsos commentarios (vel poemata) tribuit, qui sigillatim editi non fuerunt. Quid quod nostro Eratostheni, quem isti titulorum scrutatores pessime habuerant, Fellus Galeusque opera coniuncta circiter' quadraginta, alii etram sexcenta volumina assignarunt? Quis non rideat, semel auditum, Vossiụm, Sạlmasium, Fabricium commentationem in architectura versatam, et magis etiam ridiculum, eundem Fabricium medica scripta Eratosthenis posnisse? Equidem cum persuasissimum haberem, istam molestissiman licet componendi fragmenta operam summam huius quaeotionis complecti, in eam rite perscquendam onuni in. cubui studio et ardore, legenda viri hac in arte sine comparatione principis, V,alckenarii, vestigia ratus,

Ultimum obtinuit locum illustratio, fragmentorum. Hic no quis modulum normamque, ad quam singula huius opellae conformavi, temere sibi carpendam arripiat: illustrationem omnino praetermissam fuisse, ultro confiteor. Duplex datur explicandi ratio, altera indocta et puerilis, qua cum primum cuiuscunque argumenti sermo est iniectus, locos. undecunque acceptos, rei sive accommodatos sive ineptos, ingerant et convehant; altera docta, liberalis, ingenua, quae cognitione et peritia universae antiquitatis capitum suffulta, quaecunque expedienda sibi sumpsit, corum vim et sententiam, toto patefacto ambitu, originibusque ad istius scriptoris us. que aetatem accurate et peropecte repetitis, expendere norit atque investigare. Ad priorem igitur regulam multos, ad altexam ne septem quidem auctores post 
tot saeculoram desuroum indagatos esse contenderim: illam, sprevi, hanc ignoravi. Atque Eratosthenis, viri et acumine et eruditione longe praestantissimi, quis tandem et Geographica, et reliqua mathematici argumenti, et opera litterata, pauca adhuc lustra emensus, ea quae tantum decet virum amplitudine et doctrinas copia explicaverit? Satis habui viam, qua monumenta maximi. illius ingenii exstructa et adornata fuerint, paucis ea breviloquentia quam ubique effingere volui demonstrasse; idemque satis habui, allatis quae praesertim ad Geographica diudicanda pertinerent, taedio la: boris, supra quam dici potest molesti et temporis rapacissimi, tandem aliquanda emersisse: ut Eratosthenis memoria sensim evanida atque obscurata clarior et fruetuosior emineret. Quod si quis neget omnino hanc. provinciam suscipi debuisse, eidem ipse assentiar, iustam fragmentorum tractationem illius praecipue, qui ingenuam attigerit interpretationem, domiciliam esse opinans, At quot eorum omnium qui hoc in litterarum genere principatus potiti censentur eum scriptorem Graecum vel Romanum, qui operae referret pretium, nedum fragmenta, reḍintegranda sibi proposuen runt? Proinde alterius vices non adeo erant sperandac. Quibus expositis si de recentioribus quae usu veuire patuerint adminiculis verum pronunciavero iudicium, talem non existima commemarationem quem. quam in gloriolae cupidam affectationem versurum esse. Quippe adeo intelices qui Eratosthenem adverterant rem suam gesserunt, ut tam vehementer absurda super vetusto poeta vel litterato pedestris orationis auctora prolata non meminerim. Primus pro more plura Eratosthenea enumeravit Io. Meursius ad Hesychium Mile. sium (Opp, T. VII, p. 239.) et Nicomachum p. 166. Non ita meliora dedit G. I. Vossius de historicis Graecis. Insecutus est Io, Felluo, Geometra, a Graeci tamen 
sermonis peritia abhorrens, qui praefatione ad Catasterismos multa excogitavit scripta, ac nonnulla splendidarum epularum frusta apposuit: nescio qua mente eo usus instituto; paucis enim fragmentis in hac quaestione nihil effici patet. Quae Fellus erat voiumina commentus, eandem viam ingressus auxit Galeus praefatione ad alteram opuaculorum mythologicorum editionem. Uterque testes nonnisi nomine excitarat. Neque maiore potest illis praeconio Ionsius insigniri, qui de scriptoribuo hist. philos, II, 7. nibil quod alicuius foret momenti edisseruit. Istiusmodi copiis adhibitis non sane egregia praestitit Fabricius (cuius editio Harlesiana semper hic laudatur), significavit tamen sanae mentis indicia. Qui proxime scholae Alexandrinae historiam composuit, ea qua deçebat et gravitate et iudicii severitate enarratam utilitatem sane insignem praestituram, Matterum dico, sua quoque de Eratosthene proposuit, erroribus scatentia quibuscanque (T. I. p. 130 sq. $134-37$. II. p. 35. 54. 87. alibi.): pexstringere in tanta aliorum colluvie nihil attinuit. De libellis geographicis qui ad Eratosthenem pertinuerint suo loca quaedam reperientur.

\section{VITA ERATOSTHENIS.}

Vitam tanti viri eadem qua plerosque antiquitatis auctores cura docti olim grammatici amplectebantur. Unus adhnc Sumas nonnulia etiam de scriptis praestat, qui si reliqua per argumenta eundem a diligertia et ratiocinatione sese exhibuisset, prae ceteris profecto dignissimus foret, qui neglectus iaceret oorderetquo: avivo \&siǩt.

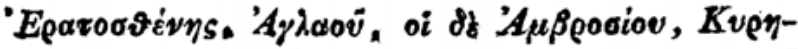

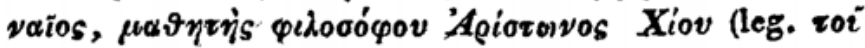

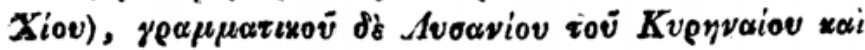




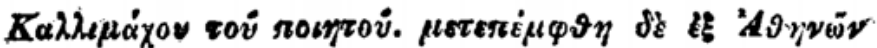

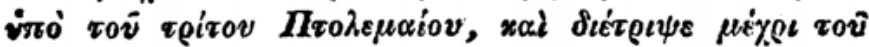

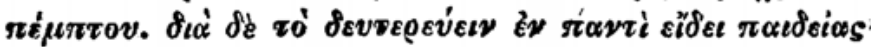

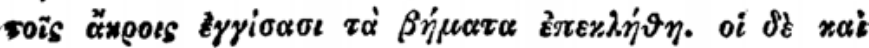

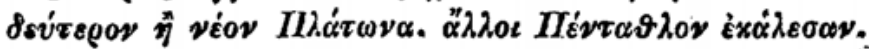

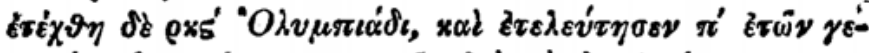

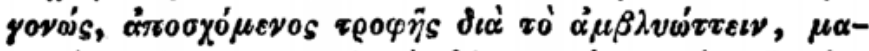

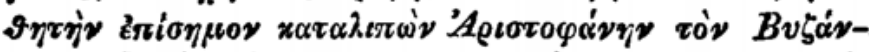

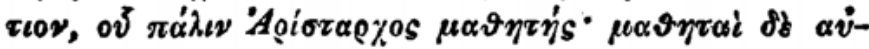

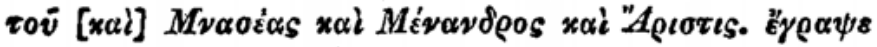

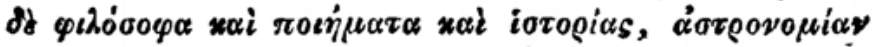

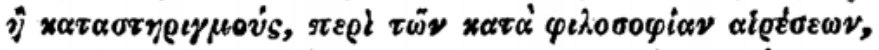

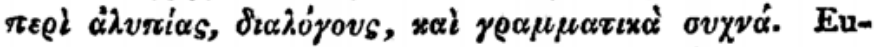
docia p. 172. locum exscribens praebet diahóyovs $\pi \lambda \varepsilon i-$

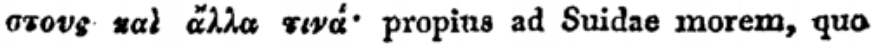
v. c. Pindaro praeter ea quae unice reliquit $\alpha \lambda \lambda . \alpha \pi \lambda_{\delta} \pi-$

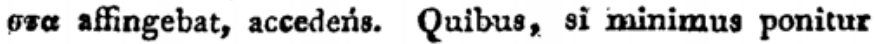
numerus, вex insunt vitia plusve minusve imperita. Quod enim primurn Eratosthenes Athenis arcessitus diitur, ad initium Geographicorum demonstrabitur, obiter tantum illic eum esse versatum. Iam vero bis, quantum Eratosthenes nobis cognoscitur, illius habitationis potuit opportunitas offerri, tum $\Delta$ ristonem et Arcesilaum cúm admodum adolescens andiret, tum quando Graeciam, fortasse Geographicis conscribendis, peragravit. Sed in hoc itinere Alexandriam adecitum fuisse, veri non oimillima verisinuilitudo est. Deinde fic' rò

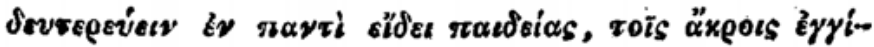

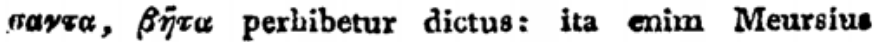
emendavit ad Hesychium Milesium, qui eandem occihit cantilenam. Accedunt Marcianus Heracl. peripli

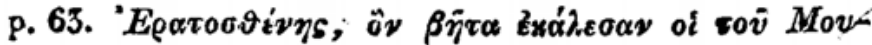

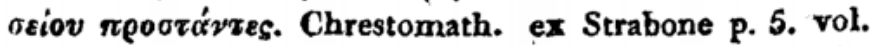

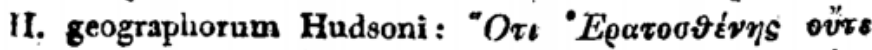

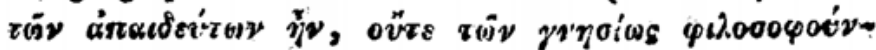




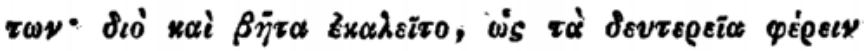

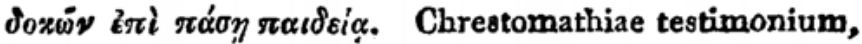
cum ista Strabonis excerpta inter se magnopere discrepent, aliunde varie interpolata, non maiorem, quam Suidae sive Hesychii, habebit vim; Marcianus autem, fraudis turpissimae convictus, cum praesertim Eratosthenis adversarium, Artemidorum, compilaverit, non iam locupletem sese testem exhibuerit. Potuerunt tamen doctissimi homines sine haesitatione Eratosthenem $\beta \ddot{j} \tau \omega$ crepare: quorum si tanti est disputationes maxime de hac voce vide apud Reinesium Var. Lectt. I. p. 31. extr. sq. Vossium de hist. Gr. p. $10 g$. Ionsium de scriptt. hist. philos. II, 7. tandem Fabricium Bibl. Gr. IV. p. 1x8. Rectius invidiae hominum cognomen tribuebat Seidelius praef. Geogr. p. XIII., quod et per se in dubium vix cadit, in tanta permultorum velis remisque pertractata contentione, indiciisque unius Strabonis de Eratosthene non intellecto adhibitis; . et oi observaris, Marcianum hanc in rem vetustissimum praestare auctorem, certissimum videbitur. Pergit Suidas : of $8 b$

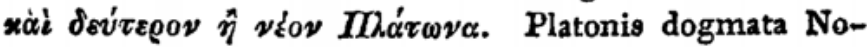
strum sectatum esse nibil admonemur; neque si dialogos ceterasve commentationes philosophicas scite Eratosthenes sollerterque, cuius indicia quaedam occurrunt, persequebatur, alter ideo Plato potuit audire; quod autem Fabricius ait IV. p. 118, propter singularem qualis fuerit etiam in Platone mathematicarum disciplinarum cum reliqua philosophia coniunctionem titulo auctum, speciosius quam verius dictum percipitur. Quapropter vel eruditi hominis sententia, honorifica lande Eratosthenem condecorantis, vel $I \lambda \alpha \alpha \tau \omega \nu$ เxou compendium Suidam fefellisse censendum est. Praeterea $\varphi$ i hóooya ante philosophica scripta memorata non eximinm produnt acumen; iovopias Eratosthenem emisisse falsum est, mendaciique in societatem venit etiain 


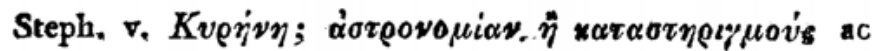

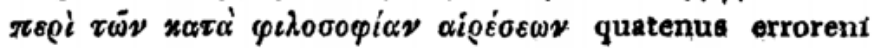
exhibeant, secuturis de Mexcurio et Philosophicis No-

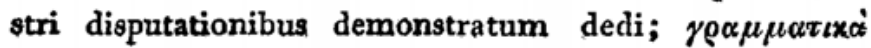
$\sigma v \chi \nu \alpha$ illinc unde pleraque Suidae emanavit. Grammaticam Eratosthenes tanquam singularem doctrinam neque exposuerat, neque quo erat ingenio exponere potnit; libros certe de antiqua comoedia neminem huc protracturum opiner. ,Eundem commisit errorem Cle-

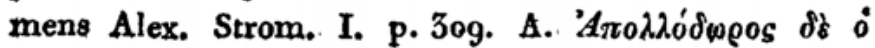

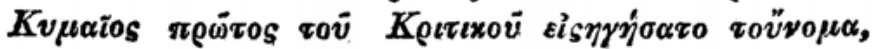

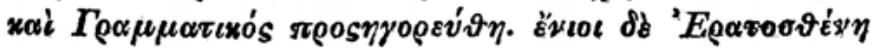

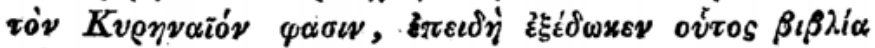

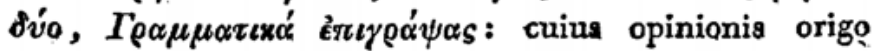
fortasse ex interpretatione temeraria significationis voü

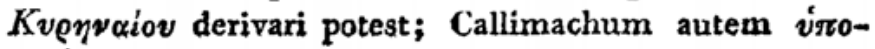

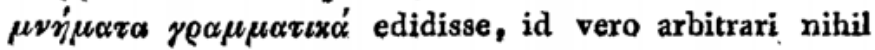
vetat, ut quem etiam Apollodorus (v. Geographicorum init.) Grammatici appellatione diserta perstrinxerit. Inter grammaticos recenset etiam Tatianus p. 107, quem vel nominasse sufficit.' Hesychium autem hac oi Diis placet Grammatica per Lexicon saepe usum fuisse, quae Felli est sententia, id tandem proferri potuisse, nemini non singularis esse imperitiae videbitur. Sed Suidae miraxe diligentiam, qui capitalia opera, Geographica et libros de antiqua comoedia, omiserit; Fabricius quidem nescio quo iure ipsum Suidam hos commentarios afferre pronunciarit Bibl. Gr. II. p. 441.

Quae igitur ad Suidam spectant vel illustrandum vel supplendum, paucis utpote pauca subiungam. Cyrenis Aglao patre Eratosthenem natum multi perhibue-

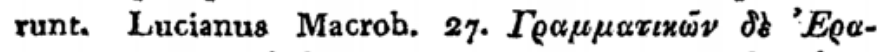

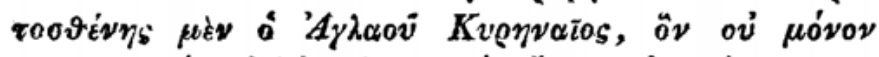

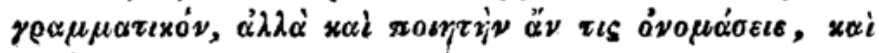

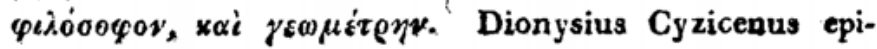


grammate infra exhibendo, Proinde Steph. Byz. v, $K v-$

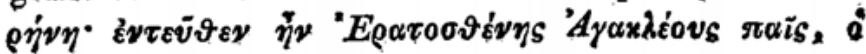

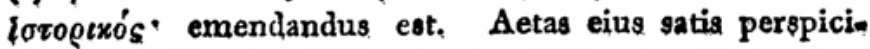
tur, tum ipsius fragmentis quibusdam (v. c. Geograph. fr, XV.), tum testimoniis, Eusebii Clironico n. MDCCCIII.

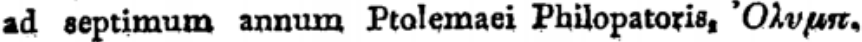

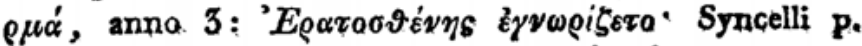
232. B. ad tempora Philippi III; qóre 'Egaroasìns

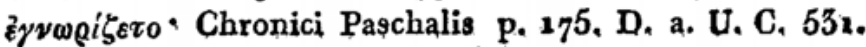

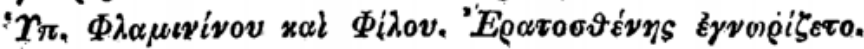
Patet hinc, Eratosthenem natu demum grandem libros vulgavisse. Alia modo florentissima eius aetas liquet fis locis, qui Archimedem cum Eratosthene coniungunt. Proclus in Euclidis Element. II. p. 20 init. da

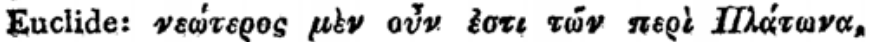

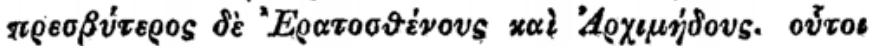

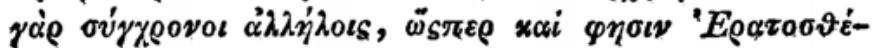
$\nu r_{j}$. Amicitiam inter utrumque intercessisse, communi geometriae amore partam firmatamqque, significat épigramma a Lessingio editum Opp. T. XIV. p. 235,

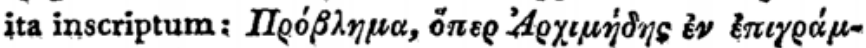

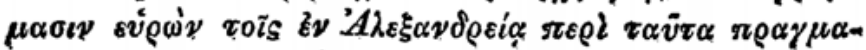

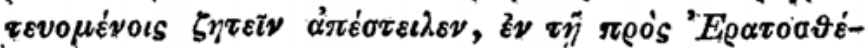

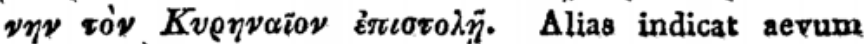

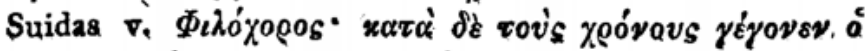

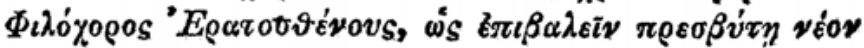

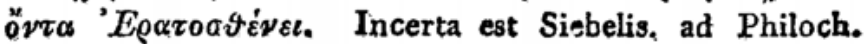
p. 3. emendatio, $\nu \varepsilon \alpha \nu i^{\prime} \nu^{\prime} E \rho \alpha \tau a \sigma \vartheta \varepsilon \dot{\varepsilon} \eta$. Lysania dicitur magistro usus fuisse, cuius memoria maxime duabus Homericorum Scholiorum notationibus (v. Wolf. proleg. p. 186.) et Athenaei XI. p. 504. B. innotuit. Callimachi disciplina oi fruebatur, vix quidquam ille ad Eratosthenis ingenium valuit: nec mirum in diversitate morum utriusque perinsigni. Quam qui perspexerit, intelliget, quanti sit faciendum Fabricia dictum III. 
p. 825, in Callimachi Airco commentatum esse sine teste edocentis: quod etiam multis antestantibus ablegandum foret. Neque vero simultatem utrumque distinuisse arbitror, declarante Callimachi fragmento, quod de Apollonio pronnnciavit (CC.): xal póvos al-

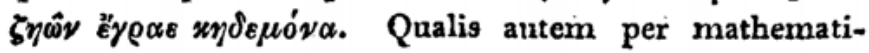
cas disciplinas dux Eratostheni affuerit, Lexicographus tradere non curavit. Iam' si Strabo, de cuins loco ad philosophica scripta agetur, verun exposuit, consuetudinem Athenis cum Zenone contraxit; in ouas tamen partes Arcesilaus et Aristo Chius pertraxerunt, quorum suctoritatem revereri ne maturior quidem aetas eum dedocuit : sed de hoc argumento affatim alibi disputatum est. Tandem a Ptolemaeo Euergeta advocatus bibliothecae Alexandrinae praefuit, in eaque urbe diem cuum obiit. Favore eum a Ptolemaeis exceptum fuisse,

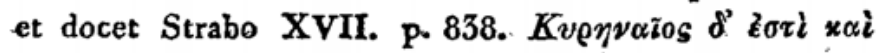

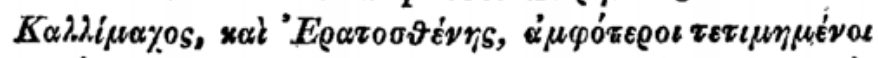

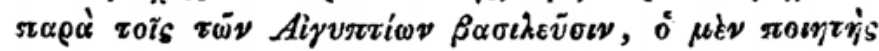

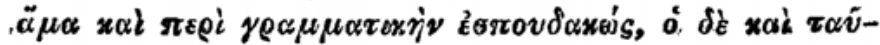

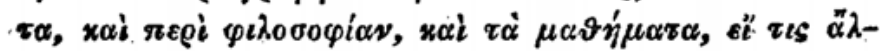

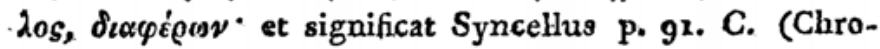

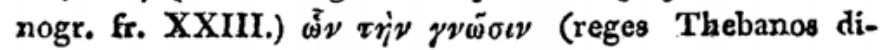

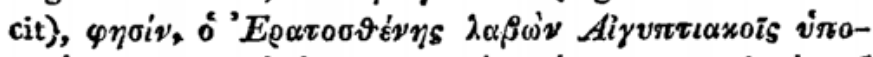

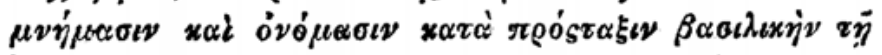

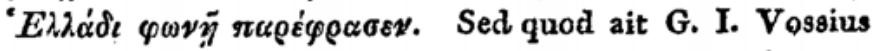
de natura artium p. 114. b. opp. tom. III., Alexandriae eins suasu armillas ac regulas ad quotidie observandos motus coelestes institutas fuisse, fortasse teste alJato fidem sibi inveniet. Octogenarium decessisse ait

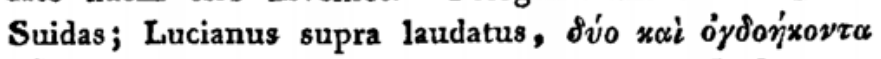

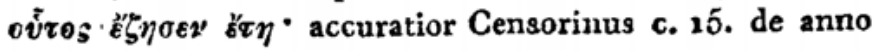
octogesimo primo sermocinans: Exatosthenes quoque, ille orbis terraxum mensor, et Xenucrates Platonicus, veteris Academiae princeps, ad eunden annum vixe- 
runt. Huius ratio, quia certae cuidam ratiocinationi. adaptatur, vera ponenda est. Quae de mortis genere Suidas protulit non improbanda, iis non adeo faver, fidemque paene abrogat Dionysii Cyziceni in Eratosthenem epigramma apnd Brunck. Analect. II. p. 255.

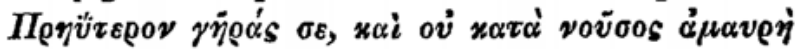

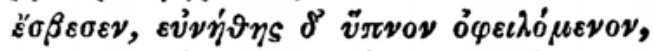

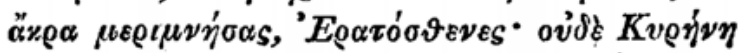

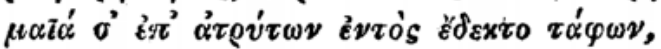

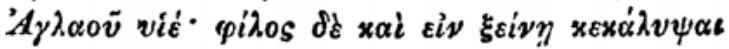

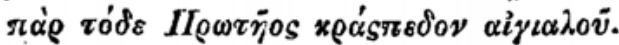

In custodia bibliothecae ei successisse Apollonium

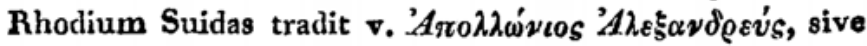
auctor vitae Apollonii. Ceterum discipnlorum Eratoothenis clarissimus est Aristophanes*), non obscurus Mnaseas; Menander autem et. Aristis, cuius nomen etiam in corruptelae suspicionem incurrit, fere delituerunt.

Omnes, quotquot viros Cyrenae insignes protulerunt, longo post se intervallo reliquit Eratosthenes, idein cunctorum, quos Ptolemaeorum favor litterarum. que amor Alexandriae unico exemplo congregavit coluitque, ex iis animi dotibus, quibus hominis docti colligitur excellentia, censuis, longe maximus. Sive enim accuratissimum requiras doctrinarum complexum, sive eruditionem minutissima quaeque adhibentem ac ouis in locis reponentem, ratione, iudicio subtilissimo,

*) $\mathrm{Ne}$ in crimen temere vocaremur, Boeckhii verba inserere visum fuit super praefat. Schol. Pindar. p. XII. monentis: Zenodoti Ephesii, Callimachi et Eratosthenis discipulus, Aristarchi magister, non de Chrysippo scripseram, sed de Aristophane Byzantio, collectis oingulis ex Suida voce. 'A nescio quo casu huc relata sunt. neponi debebant p. $\mathbf{X}_{\text {, }}$ lin. 11. post yocem qui. 
oagacitate moderatam, sive humanitatem, quae in angustiis ant anguli natura concessi aut disquisitionum minime defixa, veritatem unice investiget et ad instam ac liberalem gentis humanae sese emergat aestimationem: artiorem harum virtutum consociationem praeter Aristotelem nemo ex antiquis auctoribus Eratosthene perfectins instituisse deprehendatur. Contestantur, quae, post tantam disperditionem ac dissipationem, eius operum mathematicorum litteratornmque adhuc notitiam nimis tenuem fragmenta conservarunt; contestantur immensae olim admirationis documenta, quae etiam adversarii cum multitudine sua, tum dictis adan. xerunt. Memorabilis est titulus a Suida proditus, $\Pi \dot{\varepsilon} v-$

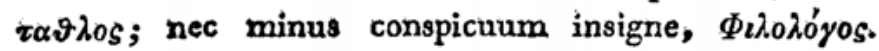
Quod enim ancipitibus Suetonii (de illustr. grammat. 10. Philologi appellationem assumpsisse videtur (Ateius), quia, sicut Eratosthenes, qui primus hoc cognomen sibi vindicavit, multiplici variaque doctrina censebatur.) verbis efficiebant, Eratosthenem sibi ipsum hoc cognomen asseruisse: refellitur notione illus vocis vetustissima, diligentissime exposita a Io. Wowerano de Polymathia c. XV., quibus notationibus adiiciantur Lobeckio observata ad Phrynich. p. 393. Litteratum enim quem Romani vocarunt id vocabuli apud personatum demum Plutarchum de poetis audiendis significavit. Sed Clementis supra adducti testimonium, secundum non-

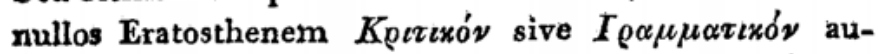
diisse, fundamento nititur perquam incerto. Critici quidem cognomentum, acrem genuinorum spuriorumque iudicem designans, potuit a libris de antiqua Comoedia peti. Clarissime tamen comparatione inter Nostrnm ceterosque Alexandxinos vel aequales adhibita Eratosthenis ingenium elucebit. In quod certamen non alii, quam Lycophro, Aratus, Callimachns, Nicander, admittentur. Sed Ly cophrone quis tandem pra: 
viorem et eruditionis et poetićae abusum manifestavit? sanam vero mentem et per Cassandram et libros de comicis frustra desideremus. Iiisdem a vitiis ne Lycophroni quidem potest $\mathrm{N}$ ic and er comparari. Minus doctrinae, plus indicii ac facultatis poeticae consignatum dedit $\Delta$ ratus carmine utroque; rectissime diudicavit Callimachus epigr. 38, tenue ac dulce dicendi genus, multis comparatum lucubrationibus, illi attribuens; reliquorum eius monnmentorum nihil amplius extat. Quos omnes superavit Call i m a ch a s, cuius praeter alios morem optime indagavit Clemena Alexandrinus: $\boldsymbol{E} \boldsymbol{v}$ -

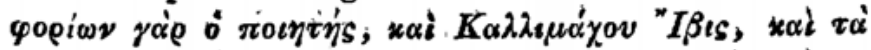

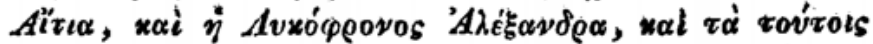

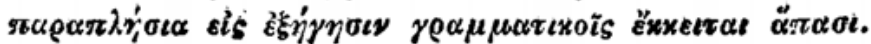
Eruditione fuit sane Callimachus onestissimus, iciemque verborum et locutionum ad modulum avium, ut recte facetissimus Sillographus, Alexandrinarum Musei andacissimus novator, qualia cum frequenter apponeret, non potuerunt non centena eins fragmenta a Grammaticis excitari : ad Eratosthenea perraro eum in ustum provocatur, unde copia reliquiarum praeceptori cedit. Sed si quid praeterea perlustratur, nihil nisi longae ac ieiunae descriptiones et excursus praesto ount, lucernam nt qui maxime redolentes. In explicanda quidem sententiarum et amoenitate et gravitate magnopere eum $\mathrm{Ph}$ iletas superasse videtur, ex cuius poematis nihil plumbei decerpitur. Iam vero exigui haec momenti existimanda prae anili fabularum dudum ab hominibus doctis excussarum tenacitate. Laudamus iudicium de Euhemero, tela hebetiora profundenti, fragmento LXXXVI., ridemus argumentum initio, ne quid ridiculi desideraretur, hymini Iovis propositum; nec quidquam stolidius afferri pottisse iis arbitramnr, quibus hymno Apollinis extremo vim illius Dei, quibus hymno in Delum Nympharum et arborum commercium vindicavit. Cetera 
hic ennmerare, quae si expenderentur, rimarum plena, ut ille ait, hac illac perpluerent, longum est.

In hac Eratosthenis praestantia nihil tamen admirabilis est iactura omnium huius operum, quorum singulare acervo inutilium antiquitatis nugarum quisque facile redimeret. Geographica enim, a multis compilata, tandem Strabonis commentariis intellectu expeditioribus succubuerunt. Alia vel difficultate argumenti perierunt, vel epitomatoribus similibusve praedonibus cesserunt, quod intelligendum imprimis de libris philo. oophicis, a Favorino praesertim usurpatis, et opusculis de antiqua Comoedia (cf. fr. II.). Neque enim multitudo iis offecit: duodecim libellis, non densis voluminibus actum de comicis fuisse veri simillimam praebet speciem. Iam cubi duplicandi methodo omissa, si tres quattuorve plilosophicae ponuntur commentationes, vix decem eius colliguntur opera, qui octoginta annos attigerit. Referenda antem videbantur sive ad genus mathematicum, cuius primaria ount Geographicorum quoque volumina, sive ad litteratos libellos. In priore versantux parte:

I. Geographica.

II. Mercurius.

III. Libri do mathematicis disciplinis.

IV. Cubi duplicatio.

sequente. V. Appendice Philosophicorum.

In altera: VI. Commentarii de antiqua Comaedia

VII. De Chronographiis. 